Supplementary Information for:

\title{
Computational Saturation Screen Reveals the Landscape of Mutations in Human Fumarate Hydratase
}

\author{
David Shorthouse ${ }^{1}$, Michael W J Hall ${ }^{2,3}$, Benjamin A Hall ${ }^{1^{*}}$ \\ 1 Department of Medical Physics and Biomedical Engineering, UCL, London, WC1E 6BT, UK \\ 2 MRC Cancer Unit, University of Cambridge, Cambridge, CB2 0XZ, UK \\ 2 Wellcome Trust Sanger Institute, Hinxton, CB10 1SA, UK
}




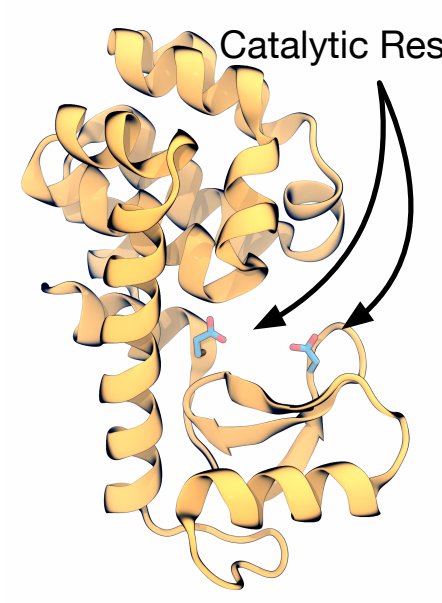

Figure S1: Structure of bacterial T4 lysozyme

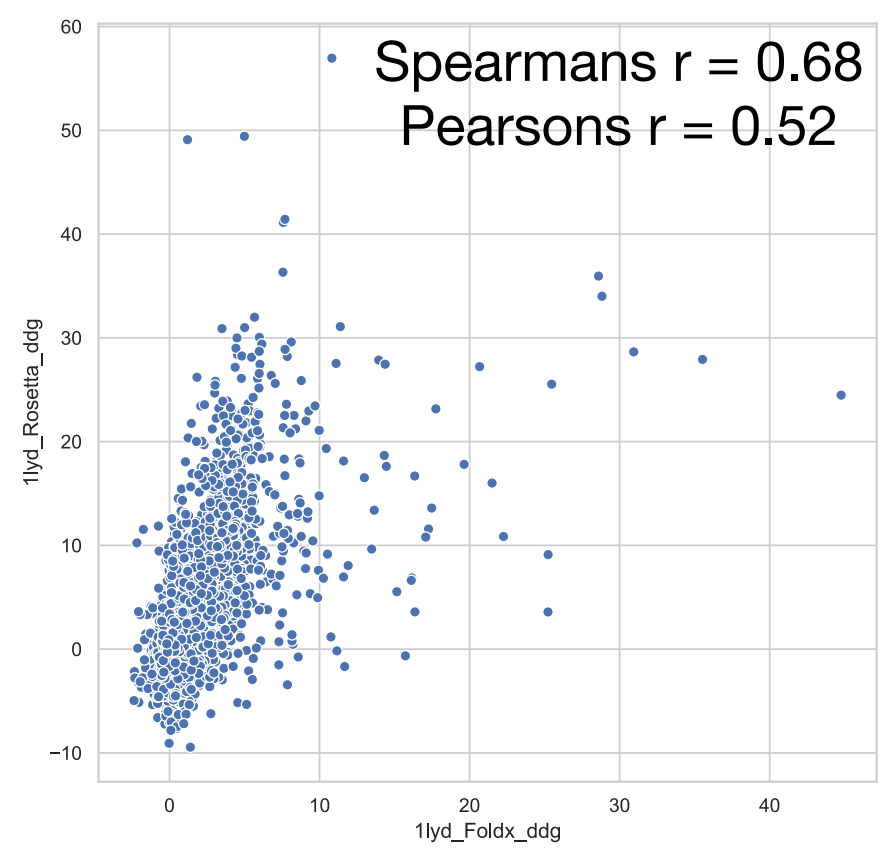

Figure S2: Spearman's rank correlation for delta-delta G calculated by rosetta and foldx for T4 lysozyme.
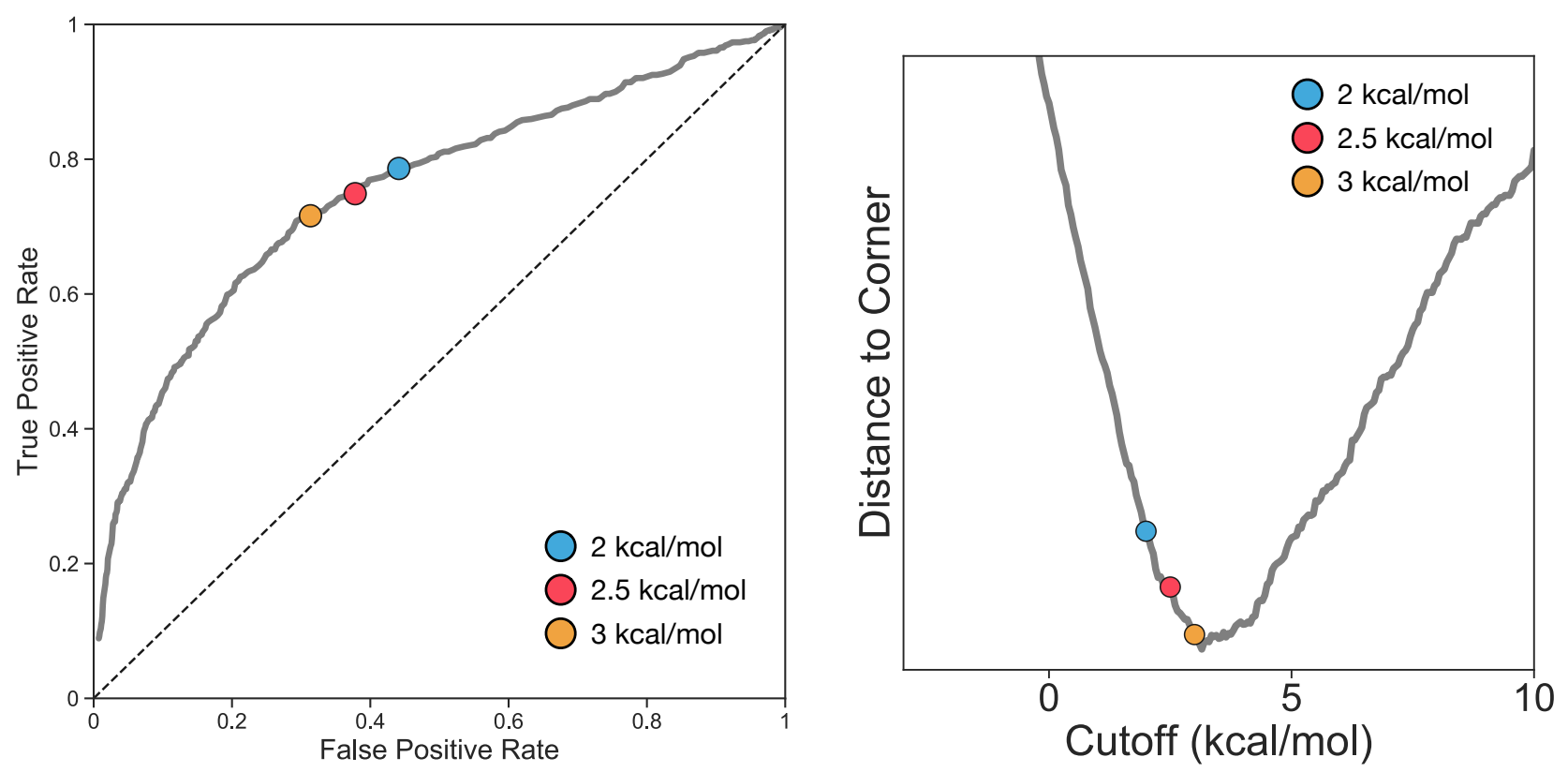

Figure S3: ROC analysis for destabilising cutoffs for measured mutations in T4 lysozyme. Left: ROC curve. Right: Distance to corner. 


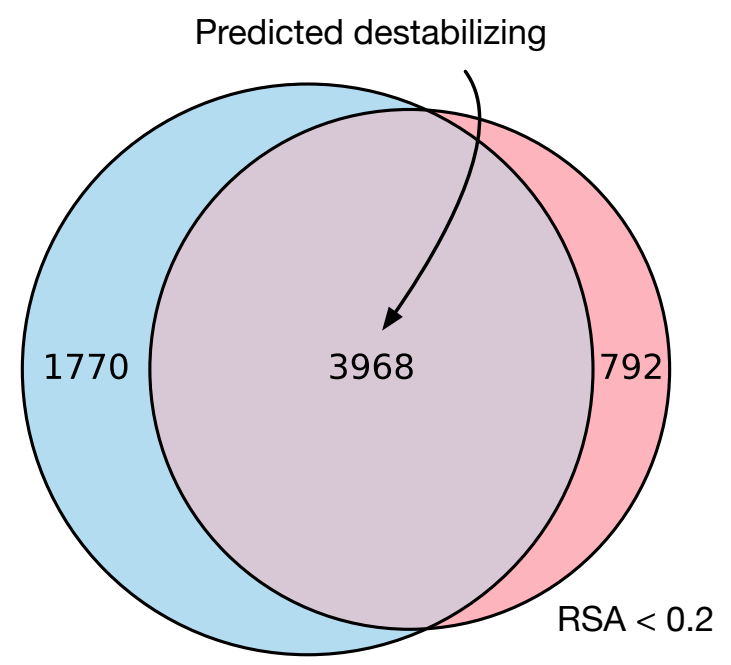

Average Energy $>2.5 \mathrm{Kcal} / \mathrm{mol}$

Figure S4: Venn showing the numbers of possible mutations in $\mathrm{FH}$ in residues with an $\mathrm{RSA}<0.2$ (red), and with a destabilising $\Delta \Delta \mathrm{G}$ (blue), resulting in predicted destabilising mutations (purple).
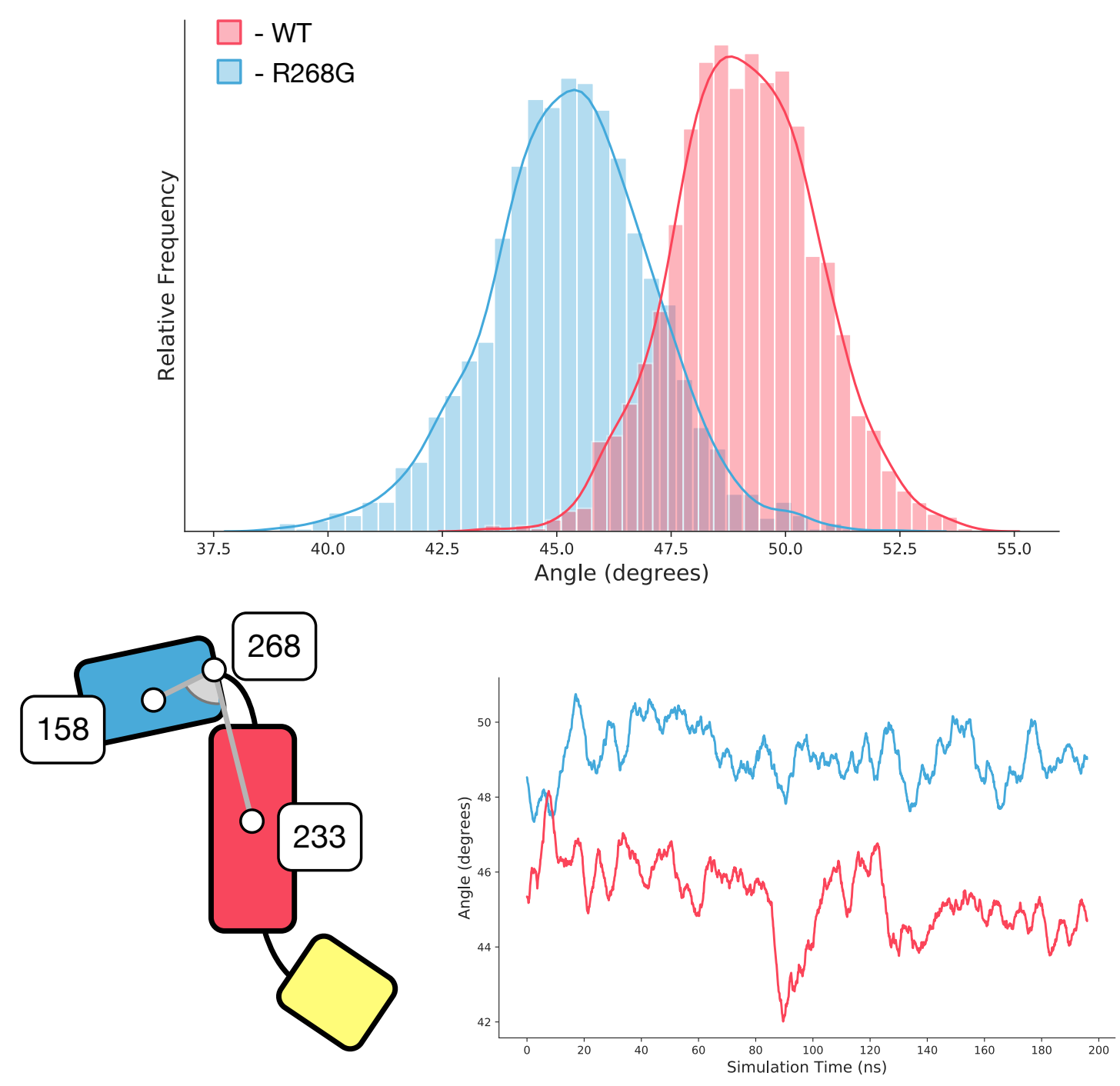

Figure S5: Molecular dynamics of WT (red) vs R268G Mutant form of FH (blue). Shown is the angle between residues 158, 268, and 331. 
Table S1: Mutational clusters for all non-benign mutations in the FH mutation database

Table S2: Classification for all possible mutations in $\mathrm{FH}$

Table S3: Classification for all mutations in T4 Lysozyme measured in Rennel et al.

Table S4: Prediction for all mutations in the FH mutation database

Table S5: Prediction for all mutations in FH in the Cancer Cell Line Encyclopedia 